\title{
Percepciones y creencias de la alimentación del niño menor de 12 meses de edad en los caseríos de la cuenca del río Momón, Iquitos-Perú
}

\author{
Perceptions and beliefs of the feeding of the child under 12 months of age in the villages of the \\ Momon river basin, Iquitos - Peru
}

José Negrón J. ${ }^{1}$; Elva Rios R. ${ }^{2}$; Paola Jorge M. ${ }^{3}$

\begin{abstract}
Resumen
El presente estudio es cualitativo y participativo, tiene como objetivo describir los cambios en las percepciones y creencias de la alimentación del niño menor de 12 meses de edad, en 15 caseríos de la cuenca del río Momón en Iquitos. Para ello, se tomó como referencia el estudio cualitativo sobre percepciones y creencias de la alimentación del niño, realizado por UNALM-ISAN y GTZ en agosto del 2002, previamente a la implementación de la campaña de comunicación sobre la alimentación de este grupo etario en dichas comunidades. Los resultados de la presente investigación, realizada en octubre del 2003, se comparan con el estudio hecho en el 2002. El recojo de la información fue realizado mediante: a) entrevistas, siendo seleccionado el informante clave por su mayor conocimiento en el tema y b) grupos focales, en los cuales se consideró a madres con niños menores de cinco años. Las guías de recolección de información fueron validadas en dos comunidades. Cada familia firmó una hoja de consentimiento, declarando contar con conocimiento previo del objetivo de la investigación y que su participación era voluntaria. Los resultados muestran que la mayoría de las madres y demás pobladores han adquirido los conocimientos sobre el inicio oportuno de la lactancia materna, las propiedades del calostro, la frecuencia de lactancia y los alimentos con los que se debe iniciar la alimentación complementaria. Sin embargo, en el caso de la frecuencia de la alimentación complementaria, se produjo una confusión con los otros mensajes que también involucraban números. Los resultados procesados fueron dados a conocer a los pobladores mediante un taller de motivación con el propósito de que lo difundieran en su comunidad.
\end{abstract}

Palabras clave: seguridad alimentaria; percepciones y creencias de la alimentación; calostro; lactancia materna; alimentación complementaria.

\begin{abstract}
This study is qualitative and participatory, and aims to describe the changes in the perceptions and beliefs concerning the feeding of the child under 12 months of age, in 15 villages of Momón River Basin in Iquitos. For this purpose we took as reference the qualitative study on perceptions and beliefs on the alimentation for infants, conducted by UNALM-ISAN and GTZ in August 2002, prior to the implementation of their communication campaign concerning proper alimentation for these age group in the mentioned communities. The results of our study, conducted in October 2003, were compared with the study done in 2002. The collection of data was conducted by: a) interviews, key informant being selected by his greater knowledge of the subject and b) focus groups, in which we considered mothers with children under five years of age. The data collection guidelines were validated in two communities. Each family signed a consent form, declaring to have prior knowledge of the purpose of the research and that their participation was voluntary. The results show that most of the mothers and other villagers have acquired knowledge about the timely initiation of breastfeeding, the properties of colostrum, the frequency of breastfeeding and food with which to start complementary feeding. However, in the case of complementary feeding frequency, there was some confusion with other messages that also involved numbers. The trial results were announced to the villagers through a motivational workshop in order to disseminate it in their community.
\end{abstract}

Keywords: food security; perceptions and beliefs about feeding; colostrum; breastfeeding; complementary feeding.

\footnotetext{
1 Escuela de Post Grado, Especialidad de Nutrición Pública. Universidad Nacional Agraria La Molina, Lima (Perú).

2 Departamento Académico de Química. Universidad Nacional Agraria La Molina, Lima (Perú). Email: erios@lamolina.edu.pe.

3 Departamento Académico de Química. Universidad Nacional Agraria La Molina, Lima (Perú). Email: paolajom@lamolina.edu.pe.
} 


\section{Introducción}

Para que los niños desarrollen su potencial genético, es decir, el máximo nivel de expresión y realización en lo físico e intelectual, se les debe garantizar la satisfacción de sus necesidades y requerimientos nutricionales, un ambiente físico apropiado y la provisión de estímulos psicológicos y sociales adecuados (Daza y Peña, 1997).

De lo anterior se desprende la importancia de asegurar una alimentación que satisfaga los requerimientos nutricionales del niño, comenzando por una lactancia materna temprana y exclusiva, seguida de una alimentación complementaria y oportuna, y la consolidación de una dieta sana a partir del primer año de vida (Daza y Peña, 1997).

Gueri y Peña (1996) y OMS/UNICEF (1999) mencionan que entre las acciones que han contribuido a mejorar el estado nutricional en el ámbito local, está la educación alimentaria nutricional, la orientación al consumidor, la promoción de la lactancia materna y la apropiada alimentación complementaria.

De otro lado, los conocimientos, aptitudes y prácticas alimentarias de las madres, determinaron en mayor medida el estado nutricional de la familia. Es así que el bajo nivel de conocimientos alimentarios y nutricionales de las madres, es uno de los factores importantes para lograr cambios apropiados en las dietas de los niños. Así mismo, las intervenciones que se realizan para promocionar mejores hábitos alimentarios que contribuyan a mejorar la nutrición resultan ser una labor muy difícil (Bado, R., Varas, N. y Medina A., 2007).

En el Perú, las prácticas de alimentación del menor de un año han mostrado cifras no alarmantes, pero sí preocupantes. En la Encuesta Nacional de Demografía y Salud Familiar (ENDES) del 2004 (INEI, 2004), se aprecia que la lactancia materna exclusiva decrece en los primeros meses de vida del niño, observándose que el $64,6 \%$ de niños de dos a tres meses, el $52,9 \%$ de cuatro a cinco meses y el $12,7 \%$ de seis a siete meses, reciben lactancia materna de manera exclusiva.

Los mensajes difundidos en la campaña de comunicación "Alimentación en el niño menor a 12 meses" han sido los siguientes:

1. Inicio inmediato de la lactancia materna al nacer: "Ni bien nace tu llullito, ahora dale tu seno. Tu leche es el único alimento que él necesita."

2. La importancia del calostro: "Mi llullito está mamando y ya me está saliendo leche amarilla" / "Esta primera leche es la más valiosa, esta protege a tu llullito de las enfermedades. No la botes, es bien buena."

3. Frecuencia de la lactancia materna: “CCuántas veces al día será bueno darle mi seno?" / "Dale bastante el seno a tu llullito por lo menos 8 veces al día o cada vez que te pida. Así vas a tener suficiente leche hasta los 6 meses."

4. La exclusividad de la lactancia materna: “¿Le puedo dar madurito o tal vez un poco de agüita?" / "Hasta los 6 meses, sólo debes darle tu seno, no necesita otro alimento más. Tu leche es el mejor alimento."

5. Inicio de la alimentación complementaria: "Si ya tiene 6 meses, ya debes de darle de comer purés nutritivos" / "Un puré nutritivo debe tener huevo o higadito o pescadito o carne de monte $\mathrm{y}$, además, echarle media cucharadita de aceite a todas sus papillas."

6. Frecuencia de los purés al día: “¿Cuántas veces al día será bueno darle puré?" / "5 veces al día debes darle comidita a tu llullito, además de tu seno."

\section{Los canales de comunicación empleados en la campaña de comunicación han sido los siguientes:}

1. Comunicación interpersonal:

- Interpersonal - personalizada: Se capacitó a los agentes comunitarios en alimentación (miembros de la comunidad que aseguraran la sostenibilidad de la transmisión del mensaje).

- Interpersonal - grupal: Se realizó 15 capacitaciones a las madres de familia (grupo meta primario) en cada comunidad.

2. Radio: Se elaboró tres tipos de mensajes radiales, a saber: los spots con 15 a 30 segundos de duración; las cuñas radiales con 15 segundos y los gingles con 2 minutos. Los mensajes se difundieron en dos emisoras locales: radios Astoria y La Voz de la Selva.

3. Materiales de apoyo: Fueron validados por el grupo meta primario durante las capacitaciones efectuadas. Estos fueron:

- Calendario

- Recetario

- Manual para el agente comunitario de alimentación

- Rotafolio

El objetivo de la presente investigación fue describir los cambios en las percepciones y las creencias entorno a la alimentación del niño menor de 12 meses de edad, en las comunidades de los caseríos de la cuenca del río Momón, en Iquitos.

\section{Materiales y métodos}

\section{Característica de la investigación}

El presente estudio es cualitativo y participativo; se realizó después de haber implementado una campaña de comunicación sobre prácticas óptimas de alimentación en el niño menor de 12 meses de edad.

Se utilizaron dos técnicas cualitativas para la obtención de la información: grupos focales y entrevistas a profundidad, que se complementan entre sí. La cantidad de las técnicas aplicadas buscaba obtener mayor validez y confiabilidad de la información. Así mismo, se contempló la participación de las personas de la comunidad en la investigación.

\section{Universo y muestra de estudio}

El universo estuvo conformado por los 15 caseríos pertenecientes al distrito de Punchana, provincia de 
Maynas, región Loreto. Estos caseríos son: Nueva York, Maynas, Almirante Guisse, Punto Alegre, Sargento Lores, San Luis de Vista Alegre, Puerto Alicia, Unión Familiar, Santo Tomas, Grau de Pampa Hermosa, Hipólito Unanue, Roca Fuerte, Flor de Agosto, Puerto Gen Gen y Centro Fuerte. Todos estos caseríos pertenecen a la zona media y alta de la cuenca del río Momón.

Para los grupos focales, se consideró a madres y padres con niños menores de cinco años. En todas las comunidades, salvo en una, no se pudo realizar los grupos focales de padres porque éstos no disponían de tiempo. Los informantes clave fueron seleccionados a partir de su mayor conocimiento del tema de estudio.

\section{Recojo y procesamiento de la información}

En agosto del 2002 se realizó una evaluación cualitativa sobre la alimentación del niño menor a 12 meses (UNALM-ISAN y GTZ, 2002), dicho estudió sirvió de contraste para discutir los resultados obtenidos luego de la campaña de comunicación "Alimentación en el niño menor a 12 meses". Por lo tanto, se realizó la comparación entre los resultados obtenidos antes de realizar dicha campaña de comunicación (entre agosto del 2002 y junio del 2003) y los resultados obtenidos en octubre del 2003. Se plantearon las siguientes variables de estudio (ver Tabla 1).

Concluido el diseño del estudio, se continuó con la confección de las guías de recolección de información las cuales fueron validadas en dos comunidades, lo que permitió corregir algunos términos y el contenido de las mismas.

Previo al recojo de información, se realizaron reuniones con las autoridades de los caseríos participantes y se explicó los objetivos y actividades que se realizarían en cada uno de los caseríos. En estas reuniones también se coordinó las fechas para la realización de las entrevistas y los grupos focales.

La metodología para el análisis fue tomada de Lovón (1996) mediante el análisis inductivo y la triangulación de datos. Se elaboraron tablas en las que las respuestas de todas las técnicas empleadas fueran agrupadas por similitud y se resaltaron aquellas que fueron mencionadas un mayor número de veces en los diferentes caseríos.

\section{Aspectos éticos}

Se explicó a cada familia el propósito de la investigación, qué se requería de ellos y se indicó que su participación sería voluntaria. En señal de conformidad firmaron una hoja de consentimiento.

Se respetó la confidencialidad y el anonimato de los informantes y, sobre todo, se buscó que los participantes hubieran recibido algún mensaje sobre la campaña de comunicación mediante uno de los canales empleado en ésta.

La devolución de la información procesada se realizó a través de los agentes comunitarios de alimentación, en un taller de motivación, con la finalidad de que lo difundieran en sus comunidades mediante la asamblea comunal que se realiza mensualmente.

\section{Resultados y discusiones}

El estudio se desarrolló entre el 10 y el 20 de octubre del 2003 y en total se realizaron 19 grupos focales y 45 entrevistas.

\section{Inicio oportuno de la lactancia}

Se recomienda a la madre que inicie la lactancia materna exclusiva dentro de la primera hora luego del parto (OMS, 2003 y Linkages, 1999a). Sin embargo, al poner esta

Tabla 1. Variables estudiadas

\begin{tabular}{|c|c|c|c|}
\hline Variables & Indicadores & Técnicas & Referencia \\
\hline $\begin{array}{l}\text { Conocimientos sobre } \\
\text { alimentación, nutrición } \\
\text { y salud }\end{array}$ & $\begin{array}{l}\text { - Conocimiento de las madres } \\
\text { sobre lactancia. } \\
\text { · Conocimiento de las } \\
\text { madres sobre alimentación } \\
\text { complementaria. }\end{array}$ & $\begin{array}{l}\text { - Entrevista } \\
\text { semiestructurada. } \\
\text { - Grupos focales. } \\
\text { - Entrevista semiestructurada. } \\
\text { - Grupos focales. }\end{array}$ & $\begin{array}{l}\text { UNICEF, } 2003 . \\
\text { UNICEF, } 2003 .\end{array}$ \\
\hline Creencias & $\begin{array}{l}\text { Creencias de las madres sobre } \\
\text { lactancia. } \\
\text { - Creencias de las madres sobre } \\
\text { alimentación complementaria. }\end{array}$ & $\begin{array}{l}\text { - Entrevista semiestructurada. } \\
\text { - Grupos focales. } \\
\text { - Entrevista semiestructurada. } \\
\text { - Grupos focales. }\end{array}$ & $\begin{array}{l}\text { UNICEF, } 2003 . \\
\text { UNICEF, } 2003 .\end{array}$ \\
\hline $\begin{array}{l}\text { Información recibida de } \\
\text { otras instituciones }\end{array}$ & $\begin{array}{l}\text { Consejos recibidos de } \\
\text { instituciones sobre lactancia } \\
\text { materna y alimentación } \\
\text { complementaria. }\end{array}$ & $\begin{array}{l}\text { Entrevista semiestructurada. } \\
\text { - Grupos focales. }\end{array}$ & $\begin{array}{l}\text { UNALM-ISAN y } \\
\text { GTZ, } 2002 .\end{array}$ \\
\hline $\begin{array}{l}\text { Información recibida } \\
\text { de los medios de } \\
\text { comunicación }\end{array}$ & $\begin{array}{l}\text { Fuente de información a } \\
\text { cerca de la lactancia materna y } \\
\text { alimentación complementaria. }\end{array}$ & $\begin{array}{l}\text { Entrevista semiestructurada. } \\
\text { - Grupos focales. }\end{array}$ & $\begin{array}{l}\text { UNALM-ISAN y } \\
\text { GTZ, } 2002 .\end{array}$ \\
\hline
\end{tabular}


recomendación en unidad de tiempo: horas o fracción, se presentan muchas interpretaciones. En la recomendación mundial de los Diez Pasos para Iniciar la Lactancia Materna Exitosa (OMS, 2010), se menciona empezar esta práctica dentro de la primera media hora después del parto.

Es así que el pronto inicio de esta práctica garantiza una lactancia exitosa (Murray, J., Newes, G., Graeff, J., Fields, R., Rasmuson, M., Salgado, R. y Sanghvi, T., 2001). Esto es debido a que la secreción de leche de la glándula mamaria depende de la estimulación de varias hormonas, entre ellas: los estrógenos, la progesterona, la prolactina y la oxitocina, estas dos últimas intervienen en el reflejo neurológico denominado "reflejo de bajada de leche". En suma, la succión del pezón por el niño y el vaciado de la glándula mamaria es el mejor estímulo para la producción de leche (OMS, 2007). Por lo tanto, el mensaje difundido en la campaña de comunicación de iniciar la lactancia materna dentro de la primera media hora luego del nacimiento, es pertinente y acorde con las recomendaciones de UNICEF (1998).

Al respecto, en el estudio cualitativo previo (UNALMISAN y GTZ, 2002), los testimonios de las madres refirieron el inicio tardío de la lactancia, lo cual ocurría hasta tres días después del parto. Esto se debía a una serie de creencias como que el seno de la madre "no se llenaba”, de que el recién nacido demora en lactar porque “no mama rápido" o la espera del "llanto" del niño como una señal de demanda de leche del recién nacido.

En el presente estudio, la mayoría de las madres mencionó el inicio oportuno de la lactancia, utilizando frases propias que indicaban temporalidad y en algunos casos se refirieron a intervalos de tiempo: "Ni bien nace..., al momento que nace..." (Grupo focal de madres de los caseríos Centro Fuerte y Flor de Agosto); “... al rato que nace, al momento que nace hay que darle chucho." (Grupo focal de madres de los caseríos Sargento Lores y Punto Alegre).

Además, la mayoría de las madres refirió haber afianzado más sus conocimientos sobre esta práctica de alimentación del niño y mencionó lo siguiente: “...darle chucho (seno) porque el niño se alimenta de esa leche..." (Grupo focal de madres de los caseríos de Centro Fuerte, Sargento Lores); “...porque el niño ya por recibir ya está de hambre, la leche le da fuerza, nutrición y vitalidad..." (Grupo focal de padres de los caseríos de Santo Tomás).

Aunque un grupo minoritario mencionó prácticas no adecuadas, esto puede deberse a que el mensaje no fue recibido por los pobladores o hubo cierta confusión con otros mensajes. Algunos comentarios recogidos: " $A$ las dos o cuatro horas..." (Grupo focal de madres del caserío Nueva York); “... a las ocho horas...” (Grupo focal de madres del caserío Grau de Pampa Hermosa).

Cuando se indagó acerca de las fuentes de información, una gran parte de los pobladores manifestó que lo habían escuchado en las capacitaciones de una campaña de comunicación de la Asociación para la Amazonía (APA) que se realizó en sus comunidades, seguido de medio impreso y por radio: "Lo escuché en la capacitación ...",
“... también está en el calendario” (La mayor parte de las comunidades). "Por experiencia, posta, hospital, centro de salud..." (Grupo focal de madres Punto Alegre y Centro Fuerte).

\section{Consumo de calostro: primera leche}

El calostro tiene un valor particular en la nutrición y salud del infante. Es considerado como la primera inmunización del niño debido a su alto contenido de proteínas y vitaminas solubles en grasa y sus propiedades anti-infecciosas (WHO, 1989), en especial frente a procesos diarreicos (OPS, 1994).

En el primer estudio (UNALM-ISAN y GTZ, 2000), la mayoría de comunidades no conocía el término calostro, pero sí lo reconocía como la "primera leche", esta era descrita como "líquido amarillo", "almidón media cremita, espesita" o también como el calostro de las vacas “palustre” y “cuajadito”. Los testimonios indicaban que las mujeres de las comunidades no les daban el calostro, lo extraían de sus senos exprimiéndolo y lo desechaban porque pensaban que no servía, debido a su aspecto, o que podía enfermar al recién nacido causándole vómitos o diarreas: "Es una leche que no vale, se ve fea ... mal aspecto." (caserío Centro Fuerte).

En el presente estudio, se observó que la mayoría identificaba el término "calostro" relacionándolo con el óptimo estado de salud del niño, una minoría agregó además los beneficios antisépticos para el recién nacido: “... es el calostro, es la vacuna (...) como la primera vacuna que recibe el niño que lo mantiene alejado de las enfermedades (...) para que el niño no tenga enfermedades, lo protege, inmuniza a la criatura." (Grupo focal de madres de los caseríos Puerto Gen Gen, Flor de Agosto y Unión Familiar). "Es el calustre, es la leche amarilla para que limpie de las enfermedades, para que les cuide de las enfermedades, de más cuidado, los protege, es el mejor alimento." (Grupos focales de madres de los caseríos Maynas, Punto Alegre, Unión Familiar y Santo Tomás).

Otro grupo minoritario continuó mencionando al calostro como la leche amarilla similar a lo encontrado en el primer estudio, en donde describieron al calostro como "líquido amarillo": "Es la leche amarilla que da vitaminas y no vale botar porque lo tiene que tomar el niño, engorda al niño." (Grupo focal de madres de Santo Tomás y Unión Familiar y grupo focal de adolescentes Centro Fuerte). Cuando se indagó sobre la fuente de información de los conocimientos aprendidos en relación al calostro, en su mayoría, manifestaron los medios empleados en la campaña de comunicación: "Calendario, radio, capacitaciones, sanitario (técnico de salud).” (Grupo focal de madres de los caseríos Santo Tomás, Unión Familiar, Punto Alegre y Maynas).

\section{Lactancia Materna Exclusiva}

La lactancia materna exclusiva durante los primeros seis meses es una recomendación de salud pública (OMS, 2007 y UNICEF, 1998) que permite cubrir los requerimientos 
nutricionales del niño, evitando la disminución de la ingesta de nutrientes, y previene la transmisión de enfermedades por contaminación de alimentos (OMS, 2007). Al respecto, en la primera evaluación cualitativa (UNALM-ISAN y GTZ, 2002), la mayoría de las madres introducía algún tipo de alimento antes del inicio de la lactancia, por ejemplo, maduro, mazamorra de plátanos, galleta, panetela, paté y leche en biberón.

Los motivos de esta práctica alimentaria por parte de las madres, eran sus percepciones con relación al hambre de su niño, creyendo que el niño "nace queriendo comer" o mencionando problemas con la producción de leche, indicando que "su seno no se llena"; además, cuando percibían que el niño nacía bajo de peso lo trataban con "leche de biberón para ayudarlo,... porque está flaquito."

En el presente estudio, después de la campaña de comunicación, los pobladores refirieron adecuados conocimientos sobre la lactancia materna exclusiva. La mayoría de las comunidades expresó lo siguiente: "El único alimento que se les da hasta los 6 meses es la leche materna (...) pura teta, pura lactancia hasta los seis meses (...), hasta los seis meses hay que darle el seno."

Asimismo, la mayoría de pobladores mencionó algunas ventajas adecuadas de la lactancia materna como fuente de sustancias nutritivas para la alimentación del infante. Por otro lado, la mayoría de los pobladores mencionó que la razón por la que se recomienda la lactancia materna durante seis meses es para disminuir los sucesos de enfermedades producidas por la introducción de otros alimentos, explicándolo de la siguiente manera:

"En la leche están las sustancias y vitaminas." (La mayoría de las comunidades).

"Porque otro alimento lo puede enfermar (...) porque todavía no come (...), es lo mejor que puede tener la madre (...) porque cuando nace de eso vive, otra cosa le hace daño (...), no debe comer porque se enferma su estomaguito." (La mayoría de las comunidades).

Con relación a la fuente de información, la mayoría de los pobladores hizo referencia a los medios de comunicación empleados en la campaña (capacitaciones y calendarios principalmente).

\section{Frecuencia de lactancia materna}

Las prácticas adecuadas de la lactancia implican que se amamante frecuentemente al niño, entre 8 a 12 veces al día, cada 2 o 3 horas, en especial en los primeros meses, debido a que el estómago del niño es pequeño y debe llenarse frecuentemente (Linkages, 1999b).

Al respecto, en la primera evaluación cualitativa (UNALM-ISAN y GTZ, 2002), las madres no tenían conocimiento adecuado sobre la frecuencia de la lactancia materna exclusiva, ya que solo en una comunidad se mencionó que se le daba 10 veces al día, lo cual estaba de acuerdo con las recomendaciones. A diferencia de las demás comunidades que mencionaron que daban de lactar la mitad de veces de lo recomendado.

En este estudio, luego de la campaña de comunicación, los pobladores manifestaron adecuados conocimientos sobre la frecuencia de la lactancia materna exclusiva. La mayoría de los testimonios hicieron referencia a un número específico de veces acorde con lo difundido en los mensajes y, en otros casos, a periodos de tiempo: "Ocho veces al día o más (...), ocho veces al día o cada vez que te pide (...) ocho a diez veces al día y si quiere más se le da." (Grupos focales de madres de los caseríos Almirante Guisse, Santo Tomás y Flor de Agosto; entrevista en profundidad a padre con niño menor de cinco años del caserío Punto Alegre). "Ocho veces, si el niño lacta cuatro veces hay que fastidiarlo para que lacte más." (Grupo focal de madres del caserío Maynas).

Una menor cantidad de pobladores mencionó que la frecuencia de la lactancia está regida por la voluntad del niño, esto es, cuando las madres reconocían ciertas manifestaciones del niño como el llanto o la palpada de seno, interpretándolas como señales de hambre, mediante las siguientes frases: "Las veces que quiera, cada vez que quiera, cuando el niño pide, cuando quiere el niño, a cada rato, cuando lloran." (Grupo focal de madres de los caseríos Puerto Gen Gen, San Luis y Sargento Lores). Asimismo, un grupo minoritario de pobladores refirió una inadecuada frecuencia de lactancia, mencionando la mitad de lo recomendado: "Cada cuatro o cinco horas (...), de cuatro a seis veces al día." (Entrevista a madre del caserío Nueva York).

Por otro lado, la mayoría de los pobladores mencionó que el propósito de la frecuencia de la lactancia materna está relacionado con la buena alimentación del niño, esta nueva información complementa los conocimientos adecuados sobre la asiduidad de la lactancia materna que tienen los pobladores, como lo muestra este testimonio "Cada vez que quiere porque de eso se alimenta hasta los seis meses." (Grupo focal de madres del caserío San Luis).

Con relación a las fuentes de información empleadas para difundir y promover los conocimientos sobre la frecuencia de lactancia materna, la mayoría de los pobladores mencionó los medios de comunicación utilizados en la campaña: "Capacitaciones, Centro de salud de Bellavista, recetario, radio y calendario." (Grupos focales de madres de los caseríos Unión Familiar y Maynas).

\section{Comidas de inicio de la alimentación complementaria}

A los seis meses se inicia la alimentación complementaria, que es la introducción progresiva de alimentos en adición a la leche materna (WHO, 1989); debido a que el crecimiento del niño se acelera, se incrementan las necesidades nutricionales y la leche materna se hace insuficiente; por tal motivo, se necesita adicionar otras fuentes de alimentos en la dieta del niño (Casanueva, E., Kaufer-Horwitz, M., Pérez, A. y Arroyo, P., 2008). Asimismo, Linkages (2002) menciona que la introducción de estos alimentos complementarios debe ser de consistencia espesa: mazamorra, purés o papillas, por su alta densidad calórica.

Al respecto, en la primera evaluación cualitativa (UNALM-ISAN y GTZ, 2002), la mayoría de las madres 
inició la alimentación complementaria con preparaciones de consistencia acuosa como "sopas, mingado de arroz, masato y leche" debido a que "ellos chupan, no mascan" por tanto estas preparaciones debían ser "suaves", "no duras" y se basaban en el hecho de que los niños no poseían dientes y, por lo tanto, no podían masticar. Además, tenían la percepción de que sus hijos no podían comer carne ni grasa, pues consideraban a estos alimentos como pesados para su digestión.

En este trabajo, la mayoría de los pobladores manifestó adecuados conocimientos sobre la preparación de alimentos complementarios para el niño, así como también sobre la lista de ingredientes a usarse, difundida en la campaña de comunicación. Estos testimonios lo confirman: "...dar puré nutritivo..." (Entrevista en profundidad a padre de familia del caserío Flor de Agosto; grupo focal de madres del caserío Roca Fuerte); "Puré de yuca, papa con huevo, aceite, carnecita o pescadito (...), puré de yuca, se echa aceite, ajino (ajinomoto), ruro, hígado o pescado." (Grupo focal de madres de los caseríos Sargento Lores, Nueva York y Punto Alegre).

Una minoría de pobladores mencionó una serie de combinaciones de alimentos poco nutritivos, lo cual no guarda relación con los mensajes difundidos y con las prácticas adecuadas en esta etapa del crecimiento. Tenemos como ejemplos: "Puré de yuca, sopa de fideos." (Grupo focal de madres del caserío Centro Fuerte); "Se le debe dar maduro, galleta..." (Entrevista a madre de familia de Nueva York).

Con relación a la fuente de información, la mayoría de los pobladores mencionó los canales usados en la campaña de comunicación (capacitaciones y materiales impresos): “... calendario, recetario, capacitación..." (Grupo focal de madres del caserío Punto Alegre y entrevista en profundidad a madre del caserío Roca Fuerte).

\section{Frecuencia de comidas en la alimentación complementaria}

Es recomendable que la frecuencia del consumo de comidas complementarias sea cinco veces al día, para compensar los pequeños volúmenes que el niño puede ingerir, dada su reducida capacidad gástrica (Casanueva et al., 2008).

Linkages (2002) señala que la frecuencia de alimentación debe ser aumentada a medida que el lactante crece, utilizando una combinación de comidas y purés. Por lo tanto, los lactantes de 6 a 8 meses de edad deben recibir de 2 a 3 veces alimentos complementarios durante el día; de 9 a 11 meses de edad deben recibir de 3 a 4 veces al día y de 12 a 24 meses de edad deben recibir de 4 a 5 veces al día. Además, puntualiza que a partir de los 12 meses de edad se debe realizar la transición a la dieta familiar.

Cabe señalar que en la primera evaluación cualitativa (UNALM-ISAN y GTZ, 2002), las madres no tenían conocimiento adecuado de la asiduidad de consumo de alimentos complementarios, ellas declararon que alimentaban a sus hijos dos veces, en pocos casos tres veces al día; lo cual no está acorde con las recomendaciones. Asimismo, indicaban que este número de veces estaba sujeto al tiempo que disponían para realizar sus actividades. Por lo tanto, un aspecto que se debe considerar en la frecuencia de la alimentación complementaria es la disponibilidad de tiempo de la madre.

En este estudio la mayoría de los pobladores evidenció adecuados conocimientos sobre la frecuencia de consumo, indicando que dan a sus niños cinco comidas al día. Además, también hicieron referencia al número y al tipo de preparaciones: "Cinco veces al día ... tres purés ... y dos mazamorras ...” (La mayoría de los caseríos). Sin embargo, una minoría de pobladores manifestó otras frecuencias de consumo al día, que no estaban en las recomendaciones. Por otro lado, se evidenció que se produjo una confusión entre el número usado para indicar la frecuencia de la lactancia materna y la frecuencia de consumo de alimentos complementarios, como en estos casos: “... dos a tres veces al día...” (Entrevista a informante clave del caserío Unión Familiar); “... hasta ocho veces..." (Entrevista a informante del caserío Almirante Guisse).

Este mensaje numérico que se empleó en la campaña, posiblemente, causó confusión. A la par, se conoce el estudio realizado por UNICEF (2003), en el cual se indica que la mayoría de las familias de Loreto presentó una frecuencia de consumo de alimentación complementaria de dos veces al día, siguiendo los patrones de la zona determinado por la escasez de recursos económicos y el trabajo de la madre en la chacra, que le imposibilita acceder a más de dos comidas.

Referente a la fuente de información, la mayoría de pobladores mencionó los canales usados en la campaña de comunicación (capacitación y materiales impresos): “... capacitación, calendario, recetario, posta de salud, el panfar...” (la mayoría de las comunidades).

\section{Consideraciones finales}

Prochasca (mencionado por Graeff, J., Elder, J. y Mills, E., 1993) considera que los cambios en el comportamiento implican un proceso que abarca cinco etapas: 1) la concientización, que es cuando la persona toma en cuenta la importancia de las buenas prácticas en la alimentación del niño, lo cual se logra si la persona conoce los beneficios y, por lo tanto, está preparada para el siguiente paso; 2) la intención de practicarlo, lo cual conlleva a la etapa siguiente; 3) la prueba de hacer esas prácticas que posteriormente pasarán a un proceso de evaluación y que llevarán a 4) la adopción de la práctica y, por último, 5) que ésta se mantenga en el tiempo. Una vez que la madre adopta el nuevo comportamiento, estará en la capacidad de recomendar esta práctica a otras personas.

Linkages (2001) menciona que la mejora de las prácticas ocurre mayormente cuando las mujeres las perciben como benéficas, factibles y socialmente aceptables. Por lo tanto, para lograr este objetivo se desarrolla una comunicación interpersonal y en grupo de discusión como una estrategia para el cambio en el comportamiento. 
Tabla 2. Comparación entre los estudios cualitativos

Estudio cualitativo inicial (Agosto 2002)

Estudio cualitativo final (Octubre 2003)

Inicio oportuno de la lactancia materna:

Inicio de la alimentación del recién nacido con otros alimentos ("no se llenaba el seno", "no mama rápido”, "llanto").

Inicio oportuno de la lactancia ("al momento que nace, ... al momento que nace hay que darle chucho”).

Consumo de calostro

Desechaban el calostro ("es una leche que no vale, se ve fea ... mal aspecto").

Identifican el término "calostro" relacionándolo con el óptimo estado de salud del niño ("... es el calostro, es la vacuna, para que el niño no tenga enfermedades, lo protege").

Lactancia materna exclusiva (LME):

LME interrumpida ("Nacen queriendo comer", "su seno no se llena").

Ventajas adecuadas de la LME como fuente de sustancias nutritivas para la alimentación del infante ( "... hasta los seis meses hay que darle leche materna, el seno”).

Frecuencia de la lactancia materna:

Pocas veces al día ofrecían lactancia (aproximadamente 6 veces al día).

Mencionaron un número específico de veces ( “... 8 a 10 veces al día y si quiere más se le da”).

Inicio de la alimentación complementaria:

Alimentos de poca densidad energética ("sopas, mingado de arroz, masato y leche" debido a que "ellos chupan, no mascan").

Los niños no pueden comer carne ni aceite, pues lo consideran pesado para su digestión.

Frecuencia de comidas:

Alimentaban a sus hijos dos veces al día para tener tiempo y desarrollar sus actividades.

Hicieron referencia tanto al número como al tipo de preparación para esta etapa (“... cinco veces al día ... tres purés y dos mazamorras”).

Fuente del mensaje:

En todos los casos mencionaron que los mensajes los recibieron de los calendarios, capacitaciones y radio. Mencionaron además a la posta de salud, al centro de salud y al hospital como emisores de mensaje de alimentación.

En la Tabla 2 se tiene la comparación entre la información recogida antes y después de aplicar la campaña de comunicación "Alimentación en el niño menor a 12 meses".

\section{Conclusiones}

Todos los mensajes referentes a la lactancia materna exclusiva y a la alimentación complementaria están basados en las recomendaciones mundiales o en las adaptaciones que se han hecho para el país.

Según los resultados obtenidos, después de implementar la campaña de comunicación "Alimentación en el niño menor a 12 meses", se observó que la mayoría de las madres y demás pobladores habían adquirido los conocimientos sobre el inicio oportuno de la lactancia materna, las propiedades del calostro, la frecuencia de la lactancia y los alimentos con los que se debe iniciar la alimentación complementaria. Sin embargo, en el caso de la frecuencia de la alimentación complementaria, se ha producido una confusión con los otros mensajes que también involucraban números, como en el caso de la frecuencia de la lactancia materna. Por lo tanto, se ve la necesidad de analizar los mensajes que hacen alusión a números para mejorar la comprensión de los mismos.

\section{Literatura citada}

Bado, R.; Varas, N. y Medina, A. 2007. Percepción materna del estado nutricional infantil de niños menores de tres años en el distrito de Ocongate, Cusco. Revista Paediatrica, 9 (2). 
Casanueva, E.; Kaufer-Horwitz, M.; Pérez, A. y Arroyo, P. 2008. Nutriología Médica. (3ra ed.). Madrid, España: Editorial Médica Panamericana S. A.

Daza, C. y Peña, M. 1997. La situación alimentaria y nutricional de los niños menores de 6 años en la región de América Latina y el Caribe en Nutrición y Alimentación del niño en los primeros años de vida. Serie Paltex, OPS.

Graeff, J.; Elder, J. y Mills, E. 1993. Communication for Health and Behavior Change: A Developing Country Perspective. The Jossey-Bass Series. Prepared under the Health Com Project, Academy for Educational Development.

Gueri, M. y Peña, M. 1996. Nutrición de la madre y del niño. En M. Gueri y M. Peña (Eds.), Acciones de salud materno infantil a nivel local: según las metas de la cumbre mundial de la infancia. OPS.

INEI. 2004. Encuesta Nacional de Demografía y Salud Familiar. ENDES Continua.

LINKAGES, Project. U.S. Agency for International Development (USAID). 1999a. Recommended feeding and dietary practices to improve infant a maternal nutrition.

LINKAGES, Project. U.S. Agency for International Development (USAID). 1999b. Prácticas recomendadas para mejorar la nutrición de los lactantes durante los primeros seis meses de vida. En Datos importantes sobre alimentación.

LINKAGES, Project. U.S. Agency for International Development (USAID). 2001. Behavior Change Communication by Breastfeeding.

LINKAGES, Project. U.S. Agency for International Development (USAID). 2002. Pautas para la alimentación complementaria adecuada de lactantes de 6 a 24 meses de edad. En Datos importantes sobre alimentación.

Lovón, M. 1996. Pautas metodológicas para la realización de estudios y evaluaciones cualitativos. Proyecto educación para la atención primaria de salud. PEAPS-UNSAC/GTZ.
Murray, J.; Newes, G.; Graeff, J.; Fields, R.; Rasmuson, M.; Salgado, R. y Sanghvi, T. 2001. Conductas de relieve en la salud materno-infantil: Enfoque sobre las conductas de las personas al cuidado de los niños para establecer programas de salud materno-infantil en el seno de las comunidades. Basics II.

Organización Mundial de la Salud (OMS). 2003. Alimentación del Niño. Documento de trabajo para la Asamblea Mundial de la Salud.

Organización Mundial de la Salud (OMS). 2007. Duración óptima de lactancia materna exclusiva. Resultado de una revisión sistemática de la OMS.

Organización Mundial de la Salud (OMS). 2010. La lactancia materna elemento clave para reducir la mortalidad infantil: Diez pasos para la lactancia materna exclusiva.

Organización Mundial de la Salud (OMS)/ Fondo de las Naciones Unidas para la Infancia (UNICEF). 1999. Nutrition Essential: A guide for health managers.

Organización Panamericana de la Salud (OPS). 1994. Manual de crecimiento y desarrollo del niño. Serie Paltex para ejecutores de salud. (2da ed.). $\mathrm{N}^{\circ} 33$.

UNALM-ISAN y GTZ. 2002. Estudio cualitativo sobre prácticas de alimentación y salud en 15 comunidades de la cuenca del Río Momón. Preparado por Nadia Chiang.

Fondo de las Naciones Unidas para la Infancia (UNICEF). 1998. La iniciativa sobre la atención: Evaluación, análisis y acción para mejorar la atención relacionada en la nutrición.

Fondo de las Naciones Unidas para la Infancia. 2003. Crecimiento y Desarrollo temprano. Prácticas y recursos.

WHO. 1989. Protecting, promoting and supporting breast feeding. 